\title{
Oncology Patient Navigation Training: Results of a Pilot Study in Nigeria
}

\author{
Runcie C.W. Chidebe ${ }^{1,2}$ - Mandi L. Pratt-Chapman ${ }^{3,4}$ (D)
}

Accepted: 27 November 2020 / Published online: 7 January 2021

(C) American Association for Cancer Education 2021

\begin{abstract}
The purpose of this pilot study was to test the efficacy of an online navigation training designed to improve trainee confidence in performing core patient navigation tasks among Nigerian nurses, patient advocates, and cancer survivors. The online training originally designed for US patient navigators was supplemented by a Nigerian health system interactive session. Nurses, advocates, and cancer survivors $(n=36)$ were recruited to take a patient navigation training from May-June 2020 that consisted of approximately $10 \mathrm{~h}$ of online content and a 2 -h online session. Trainees enrolled in the no-cost training and completed all 20 lessons. Post-intervention interviews $(n=10)$ were conducted to garner feedback from trainees. Trainees reported statistically significant improvements in confidence in performing core competencies for cancer patient navigation across all lessons. Feedback was generally positive with trainees expressing satisfaction and appreciation for the training. Challenges included lack of free, broadband Internet access leading to data streaming costs on the part of trainees. Trainees who were oncology nurses indicated that funding, time, and staffing constraints could impede implementation of patient navigation in practice; but these trainees also expressed new ideas for patient support and advocacy to alleviate some barriers to care for their patients as a result of the training. This study provided preliminary data that supports the feasibility and utility of using the GW Cancer Center online patient navigation training in non-US settings. Small adaptations to training content and technological delivery could improve access to the training experience. Implementation science approaches are needed to inform sustainable patient navigation in lowresource settings.
\end{abstract}

Keywords Cancer control $\cdot$ Africa $\cdot$ Professional development $\cdot$ Education $\cdot$ Patient navigation

\section{Introduction}

Patient navigation was introduced in the USA as a strategy to bridge cancer staging and mortality differences in Harlem, New York, in the 1990s with remarkable success: 5-year survival for poor, black breast cancer patients increased from 39 to $70 \%[1,2]$. As a result of this success, patient navigation

Mandi L. Pratt-Chapman

mandi@gwu.edu

1 Project PINK BLUE - Health \& Psychological Trust Centre, Abuja, Nigeria

2 Faculty of Health, Education \& Life Sciences, Birmingham City University, Birmingham, UK

3 School of Medicine and Health Sciences, Clinical Research and Leadership, The George Washington University, Washington, DC, USA

4 The GW Cancer Center, 2600 Virginia Ave, \#300, Washington, DC 20037, USA has grown both in the USA and globally in the last two decades. However, scaling navigation to low-resource settings across the globe is challenging. There is a critical need for clinical infrastructure, workforce training, and ongoing improvements to cancer care to make navigation efforts yield optimal outcomes for cancer patients globally. Additionally, patient navigators need to be trained in foundational skills, scope of practice, and context-specific realities to serve effectively in global settings. Patient navigation at its core focuses on assessing and addressing barriers to healthcare services. Therefore, navigators need to have an adequately skilled and available healthcare provider workforce as well as sufficient resources to refer patients to when barriers to care are identified.

With 115,950 new cases of cancer and 70,327 cancer deaths in 2018, Nigeria contributes significantly to the overall cancer burden in Africa [3] and strains the healthcare system [4]. Access to cancer screenings, like mammography for eligible women, is limited because - similar to the USA - there is no population-based national cancer screening in Nigeria 
[5]. Unlike in the UK where eligible women aged 50 to 70 are invited once every 3 years through the national cancer screenings $[6,7]$, cancer screening in Nigeria is initiated by cancer non-governmental organizations like Project PINK BLUE hosted in communities as medical missions.

For a population of 202 million, only $15.5 \%$ have access to a comprehensive cancer center within $1 \mathrm{~h}$ of travel, and $31.9 \%$ cannot access care without traveling $4 \mathrm{~h}$ [8]. Navigating the complexities of the healthcare system are exacerbated by low perceptions of cancer risk [9], belief that cancer is terminal [9], societal discrimination toward cancer survivors [9], lack of insurance coverage [10], and-for cervical cancer, in particular - cultural obligations of modesty [11]. To reduce these barriers, Project PINK BLUE established the first patient navigation program in 2015 and provided an in-person navigation training to nurses, cancer survivors, social workers, and retired midwives in 2016 [12]. Trained patient navigators use mobile phone, a web-based geographical map, and their knowledge of the healthcare system to guide and support cancer patients through diagnosis, treatment, and survivorship. Navigators help to improve cancer patient adherence to recommended care by addressing psychosocial and financial barriers and explaining the complexities of cancer treatment in terms patients can understand.

To sustain the progress of patient navigation in Nigeria, continuous training is needed. However, due to the COVID19 pandemic and resource constraints, face-to-face training is currently impossible. The purpose of this pilot study was to test the efficacy of an online evidence-based patient navigation training designed for US patient navigators to improve trainee confidence in performing core patient navigation tasks among Nigerian nurses and cancer survivors. The training was supplemented by Nigerian-specific data on cancer control and navigating the local healthcare system.

\section{Participants and Methods}

\section{Participant Sample}

Nurses, advocates, and cancer survivors $(n=36)$ were recruited by Project PINK BLUE and the National Hospital Abuja School of Post-Basic Oncology Nursing to take a navigation training from May to June 2020 that consisted of approximately $10 \mathrm{~h}$ of online content and a 2 -h virtual interactive session. Trainees attended from both the northern and southern part of the country. Most participants were in training to become oncology nurses; however, some trainees were cancer survivors and staff at Project PINK BLUE. For participant characteristics, see Table 1. Of those recruited, only five participants indicated prior training of any kind related to patient navigation.
Table 1 Participant characteristics $(n=36)$

\begin{tabular}{|c|c|}
\hline Characteristic & Number (\%) \\
\hline \multicolumn{2}{|l|}{ Gender } \\
\hline Male & $5(13.9)$ \\
\hline Female & $31(86.1$ \\
\hline \multicolumn{2}{|l|}{ Age } \\
\hline $21-29$ & $3(8.3)$ \\
\hline $30-39$ & $11(30.6)$ \\
\hline $40-49$ & $18(50.0)$ \\
\hline $50-59$ & $4(11.1)$ \\
\hline \multicolumn{2}{|l|}{ Profession } \\
\hline Healthcare administrator & $2(5.6)$ \\
\hline Nurse & $19(52.8)$ \\
\hline Nurse navigator & $1(2.8)$ \\
\hline Nurse practitioner & $3(8.3)$ \\
\hline Patient navigator & $4(11.1)$ \\
\hline Physician & $1(2.8)$ \\
\hline Social worker & $3(8.3)$ \\
\hline Patient advocate & $1(2.8)$ \\
\hline Psychologist & $1(2.8)$ \\
\hline Student nurse & $1(2.8)$ \\
\hline \multicolumn{2}{|l|}{ Practice specialty } \\
\hline Internal medicine & $1(2.8)$ \\
\hline Family medicine & $1(2.8)$ \\
\hline Geriatrics & $1(2.8)$ \\
\hline Gynecology & $2(5.6)$ \\
\hline Oncology & $26(72.2)$ \\
\hline Not applicable & $4(11.1)$ \\
\hline Missing & $1(2.8)$ \\
\hline \multicolumn{2}{|l|}{ Site of practice } \\
\hline Office practice & $1(2.8)$ \\
\hline Hospital & $21(58.3)$ \\
\hline Outpatient cancer center & $9(25.0)$ \\
\hline Community health center & $1(2.8)$ \\
\hline Other & $4(11.1)$ \\
\hline \multicolumn{2}{|l|}{ Urbanicity } \\
\hline Rural & $2(5.6)$ \\
\hline Suburban & $7(19.4)$ \\
\hline Urban & $24(66.7)$ \\
\hline Unsure & $3(8.3)$ \\
\hline
\end{tabular}

\section{Intervention}

Trainees enrolled in the no-cost, GW Cancer Center Oncology Patient Navigator: The Fundamentals and completed all 20 lessons. The online course is a competency-driven training that has shown efficacy in improving learner confidence in a wide range of core duties performed by patient navigators. A recent study of US-based learners $(n=671)$ showed statistically significant learner improvements to confidence across all learning objectives 
with approximately $88 \%$ of learners planning to use their new skills in navigation practice [13]. Since the training was developed with US patient navigators in mind, trainees for this study were also invited to participate in a 2-h interactive session on June 11, 2020, that reviewed the healthcare system and state of cancer control in Nigeria.

\section{Data Collection}

Trainees were required to complete pre- and posttests that asked about confidence in performing 97 learning objectives across 20 lessons that aligned with consensus-based core competencies of patient navigators [14]. These data were captured through the GW Cancer Center's Learning Management System (LMS). Semi-structured interviews were conducted with a subset of trainees $(n=10)$ for 10-20 min and recorded through a mobile telephone. Trainees were asked open-ended questions about their experiences with the hybrid training, including what they liked most, what could be improved, and what practice changes they would incorporate after training.

\section{Data Analysis}

Quantitative data from the LMS was exported into Excel where data was cleaned and numerically coded. A codebook was created to explain how numerical codes aligned with answer options from the LMS. Excel data were uploaded into SPSS 26 for analysis. The 97 learning objectives were clustered by lesson, resulting in 20 aggregate pretest and 20 aggregate posttest scores with varying ranges due to varying number of questions asked for each lesson. Paired $t$ tests were conducted to compare preand posttest means for the 20 lessons (see Table 2). Statistical significance was set to $p<.05$ with $\alpha=.05$ and $1-\beta=.95$.

Qualitative data were transcribed verbatim, and references to participant names were removed for confidentiality. We conducted inductive coding for emergent themes.

\section{Results}

For the quantitative component of the study, trainees reported statistically significant improvements in confidence in performing core competencies for cancer patient navigation across all lessons. Results of overarching competence for each lesson showed statistically significant improvements to learner confidence in ability to improve patient navigation processes through continual self-evaluation and quality improvement $(p=.032)$ and ability to respond to the larger context and system of healthcare and call effectively on other resources in the system to provide optimal healthcare $(p=.044)$ (see Table 3). No other statistically significant effects were found.

Qualitative feedback was generally positive. However, some trainees indicated a need for additional education on cancer prevention, detection, and treatment. Many trainees did not have previous knowledge of patient navigation before the training. Despite having started post-basic nursing oncology training at the National Hospital Abuja, many trainees indicated that this was their first-time hearing about patient navigation or receiving any type of navigation training. The following themes emerged.

\section{Factors Affecting the Institutionalization of Patient Navigation}

While many of the trainees appreciated patient navigation as a helpful intervention, trainees expressed concerns about the capacity of institutions to implement and sustain patient navigation in their hospitals. One trainee indicated:

[T]o be able to get acceptance of patient navigation in our hospital community and also [to] get sponsorship, whether private partnership, or even government to make patient navigation part of hospital setting, it will be fantastic. Oncology nurse

While the concept of patient navigation was well received by the trainees as an intervention to reduce barriers and increase adherence to cancer treatment among patients, the success of patient navigation in practice will be dependent on funding for the program.

Another challenge to institutionalizing patient navigation was lack of comprehensive services to refer patients to, as well as time demands on trainees:

We don't have an oncology center presently, but we have a radiation oncologist attached with some local medical doctors. I am in an oncology department giving chemotherapy, but we don't have a standard ward so I can't say I can use my experience of patient navigation on them properly now. Maybe as time goes on, but I have plans of doing that. Oncology nurse

The training helped oncology nurses already in practice gain new skills, but application of those skills may be limited without clinical and supportive care services to refer to.

\section{Impact of the Patient Navigation Training on Oncology Nursing Practice}

One of the goals of this learning intervention was to assess whether skills learned could be put into practice among trainees. Our qualitative study suggests that trainees gained inspiration for new ways to support patients after taking the training:

So, going through this course, I really think of... setting up a support group for the patients and also soliciting for 
Table 2 Change in learner confidence in lesson objectives $(n=36, \mathrm{df}=34)$, [range]

\begin{tabular}{lcccc}
\hline Lesson & Pretest M (SD) & $\begin{array}{l}\text { Posttest M } \\
(\text { SD })\end{array}$ & $t$ & $p$ \\
& & & \\
\hline Overview of Patient Navigation \& Competencies & $15.444(3.902)$ & $17.611(2.578)$ & -3.409 & .002 \\
$\quad$ [0-20] & & & & \\
Medical Terminology [0-12] & $9.389(2.406)$ & $10.833(1.320)$ & -4.320 & $<.001$ \\
Cancer Basics [0-20] & $15.472(3.722)$ & $18.028(2.021)$ & -5.178 & $<.001$ \\
Clinical Trials [0-16] & $11.333(3.906)$ & $14.306(1.527)$ & -5.282 & $<.001$ \\
Impact of Cancer [0-4] & $3.028(.941)$ & $3.722(.454)$ & -4.868 & $<.001$ \\
U.S. Health Care System [0-16] & $11.417(3.589)$ & $14.472(1.682)$ & -5.717 & $<.001$ \\
Health Care Payment and Financing [0-12] & $7.778(3.287)$ & $10.278(1.427)$ & -5.646 & $<.001$ \\
The Role of the Patient Navigator [0-8] & $5.444(2.117)$ & $6.806(.920)$ & -4.050 & $<.001$ \\
Navigating Patients [0-24] & $16.139(5.514)$ & $20.333(2.563)$ & -5.312 & $<.001$ \\
Shared Decision-Making [0-24] & $16.417(5.320)$ & $20.361(2.451)$ & -5.492 & $<.001$ \\
Identifying Resources [0-16] & $10.361(3.766)$ & $13.472(1.748)$ & -5.683 & $<.001$ \\
Communicating with Patients [0-20] & $14.222(4.323)$ & $17.306(2.266)$ & -5.654 & $<.001$ \\
Patient Advocacy [0-32] & $20.806(7.738)$ & $27.917(3.722)$ & -7.124 & $<.001$ \\
Culturally Competent Communication [0-24] & $15.528(5.794)$ & $20.111(3.069)$ & -6.320 & $<.001$ \\
Scope of Practice [0-24] & $15.778(5.836)$ & $20.361(3.044)$ & -5.808 & $<.001$ \\
Ethics and Patient Rights [0-24] & $15.833(5.789)$ & $20.778(2.789)$ & -6.334 & $<.001$ \\
Practicing Efficiently and Effectively [0-24] & $16.000(5.826)$ & $20.667(2.438)$ & -5.623 & $<.001$ \\
Health Care Team Collaboration [0-20] & $14.028(4.843)$ & $17.222(2.166)$ & -4.498 & $<.001$ \\
Program Evaluation and Quality Improvement & $15.833(6.300)$ & $20.417(2.430)$ & -4.922 & $<.001$ \\
$\quad$ 0-24] & & & & \\
Personal and Professional Development [0-24] & $16.028(6.162)$ & $20.889(2.734)$ & -6.177 & $<.001$ \\
\hline & & &
\end{tabular}

Italics represents statistically significance at $p<.05$ funds for them. Because in our center we have not started that, and the patients are really suffering...Oncology nurse

As a result of the training, another respondent indicated a new commitment to identifying financial resources and advocating for patients in need:

You know, some of them [patients] do not even have money. They have financial constraint to continue [treatment]. But through the training, I got to understand that... [I] can lead patient to other people [resources] that can help them. And I have made up my mind to be an advocate, to connect them to support system that will help them in their treatment. Oncology nurse

Identifying financial supports and educating patients and families on where to look for resources are critical given high outof-pocket costs.

\section{Need for Additional Staffing Focused on Patient Navigation}

A few trainees who are cancer patients and staff at Project PINK BLUE expressed improved capacity to navigate patients and follow up with them. However, trainees who were oncology nurses expressed concern that their existing clinical workload would limit their ability to focus on navigation. Nevertheless, a renewed commitment to ongoing patient follow-up to improve adherence to care emerged as an important theme:

Even though, my profession [nursing] encouraged follow up, but because of the workload, sometimes you may not really follow-up patients the way it is supposed to be... With this training of patient navigation, I was really encouraged and energized to follow up my patient because starting is not the issue but how they finish. And most of the patients start treatment without finishing and they will end up with complication. But if you cultivate the habit of following up patients, it will really go a long way assisting the patient to cure or to find solutions to the problem. Oncology nurse

In cancer hospitals in Nigeria, oncology nurses are paid for their role as oncology nurses, not for navigating patients. Hence, there is a need for additional, dedicated patient navigators to focus on assessing barriers to care and following up with patients if a navigation program is to succeed.

\section{Adaptations Needed for International Learners}

The patient navigation training was supposed to be in two phases, online and face-to-face training. With COVID-19 
Table 3 Learner confidence in overarching competencies $(n=$ 36 , df $=34)$, range $[0-4]$

\begin{tabular}{|c|c|c|c|c|}
\hline Overarching competencies & $\begin{array}{l}\text { Pretest M } \\
\text { (SD) }\end{array}$ & $\begin{array}{l}\text { Posttest M } \\
\text { (SD) }\end{array}$ & $t$ & $p$ \\
\hline $\begin{array}{l}\text { I am confident in my ability to facilitate patient-centered care } \\
\text { that is compassionate, appropriate, and effective for the } \\
\text { treatment of cancer and the promotion of health }\end{array}$ & $3.34(.873)$ & $3.54(.505)$ & -1.125 & 0.268 \\
\hline $\begin{array}{l}\text { I am confident in my ability to demonstrate basic } \\
\text { understanding of cancer, healthcare systems, and how } \\
\text { patients access care and services across the cancer } \\
\text { continuum to support and assist patients }\end{array}$ & $3.37(.770)$ & $3.49(.562)$ & -0.780 & 0.441 \\
\hline $\begin{array}{l}\text { I am confident in my ability to improve patient navigation } \\
\text { processes through continual self-evaluation and quality } \\
\text { improvement and promote and advance the profession }\end{array}$ & $3.17(.891)$ & $3.51(.507)$ & -2.095 & 0.044 \\
\hline $\begin{array}{l}\text { I am confident in my ability to demonstrate interpersonal and } \\
\text { communication skills that result in the effective exchange } \\
\text { of information and collaboration with patients, their } \\
\text { families, and health professionals }\end{array}$ & $3.51(.781)$ & $3.54(.505)$ & -0.172 & 0.865 \\
\hline $\begin{array}{l}\text { I am confident in my ability to demonstrate a commitment to } \\
\text { carrying out professional responsibilities and an adherence } \\
\text { to ethical principles }\end{array}$ & $3.31(.900)$ & $3.63(.490)$ & -1.769 & 0.086 \\
\hline $\begin{array}{l}\text { I am confident in my ability to demonstrate an awareness of } \\
\text { and responsiveness to the larger context and system of } \\
\text { healthcare, as well as the ability to call effectively on other } \\
\text { resources in the system to provide optimal healthcare }\end{array}$ & $3.11(.718)$ & $3.46(.561)$ & -2.240 & 0.032 \\
\hline $\begin{array}{l}\text { I am confident in my ability to demonstrate ability to engage } \\
\text { in an interprofessional team in a manner that optimizes safe, } \\
\text { effective patient- and population-centered care }\end{array}$ & $3.29(.926)$ & $3.54(.505)$ & -1.358 & 0.183 \\
\hline $\begin{array}{l}\text { I am confident in my ability to demonstrate qualities required } \\
\text { to sustain lifelong personal and professional growth }\end{array}$ & $3.37(.877)$ & $3.46(.780)$ & -0.393 & 0.697 \\
\hline
\end{tabular}

Italics represents statistically significance at $p<.05$ pandemic, the face-to-face part of the training was canceled to ensure social distancing as recommended by public health authorities. Hence, trainees were asked about challenges in using an online training platform. Some respondents expressed that poor Internet connectivity, cost of mobile data, and lack of reliable power were challenges to completing the online training. Other trainees expressed problems with training videos uploading slowly or not at all and taking up a great deal of data.

What I don't just like... is the video aspect. At time you want to open it, it will not open. And at times it [takes] long [time] that you will be consuming a lot of data. I spent a lot of money on buying [mobile] data to do the [online training] but I gained a lot from it... Maybe the video is something that we can download that maybe you can watch it offline... Oncology nurse

Another challenge reported by trainees was the limited relevance of some content. For example, while the US healthcare system module was optional, most trainees wished to take all of the modules, leading to frustration regarding its limited relevance of US-specific content.

[A] challenge [for me] was having to marry the [U.S.] system of healthcare described by George Washington
University into the Nigerian [healthcare system...I was trying to you know, look at it from the Nigerian perspective... Oncology nurse

Despite these challenges and limitations, the same trainee indicated: "But the course was awesome."

\section{Discussion}

The Oncology Patient Navigator: The Fundamentals was created with US-based patient navigators in mind as learners. Despite the lack of tailored content, a cohort of Nigerian patient navigators showed improvement across all learning objectives and lessons (Table 2). It should be noted that when asked about specific ability to call upon skills, definitions, approaches, or strategies at the learning objective level, learners showed statistically significant improvements from pretest to posttest. However, when asked about confidence in overarching competence for each lesson, greatest improvements to confidence were in abilities to improve navigation processes and call upon resources within the larger healthcare context (Table 3). The lack of statistical significance for other high-level competencies may be due to high agreement in capabilities in performing the competency at pretest, leaving little room for improvement. 
Feedback from the qualitative arm of this study suggests that the online patient navigation will be more beneficial to patient navigators in non-US settings if resources were downloadable to allow for less data usage and to provide greater flexibility to trainees to watch videos at a convenient time. In this study, Project PINK BLUE hosted an online session on Nigeria's healthcare system to replace the US healthcare system module. Providing an archive of context-specific modules for trainees to access on demand may also be helpful to future trainees.

A major finding of this pilot study is that oncology nurses, cancer survivors, and nonprofit staff are interested in, appreciative of, and benefit from patient navigation training. However, there is a need to consider implementation factors to optimize institutionalization of patient navigation in practice within cancer centers and hospitals in low-resource contexts. For example, in Nigeria, less than 5\% of the population is covered by health insurance [15]. While Nigeria's National Health Insurance Scheme (NHIS) started partial insurance coverage for cancer care as a result of advocacy efforts on the part of Project PINK BLUE and Network of People Impacted by Cancer in Nigeria (NePICiN) in 2019, at the time of this writing, there is no clarity on the specific essential cancer medicine that will be covered by the insurance scheme. This policy and coverage issue provides a local opportunity for navigators to advocate on behalf of patients in financial need. Theory-driven implementation science approaches could help to highlight such context-specific factors to patient navigation implementation and sustainment. Such approaches could significantly improve the quality of cancer care in Nigeria and create new employment opportunities for trained navigators [16].

\section{Conclusion}

The GW Cancer Center Oncology Patient Navigator: The Fundamentals was created with US-based patient navigators in mind; however, results from this study suggest that the training can be helpful to patient navigators in other settings. The primary limitation of this study was the limited relevance of US-specific content in the training. An unanticipated challenge was out-of-pocket costs of trainees needing to stream video content in settings without broadband Internet access. Overall, this study yielded preliminary data to support the efficacy of the training in non-US contexts while providing important suggestions for adaptations needed for low-resource settings. Future research should examine implementation factors that support sustainable navigation programs in Nigeria as well as technological adjustments to alleviate data costs and the frustration of slow downloading files. In the meantime, this no-cost training may be used in tandem with tailored, local healthcare and financing information to provide a strong foundation for patient navigators across the globe.
Acknowledgments The authors would like to thank Dr. Vitalis Okwor and Mampak Nanre who helped with recruitment of trainees as well as Chinonso Ipiankama who helped in data collection.

Authors' Contributions MPC created the learning intervention, led the writing, and conducted the quantitative analysis. RC conceptualized delivery of the intervention to navigators in Nigeria, contributed to the writing, and conducted the qualitative analysis. Both authors approved the final manuscript.

Funding The corresponding author's time creating the original intervention was funded by the Centers for Disease Control and Prevention (CDC) award \#1U38DP004972-01. The results represent the authors' views and not necessarily those of the CDC. Results have not been reported elsewhere.

Data Availability Data requests can be made to the corresponding author.

\section{Compliance with Ethical Standards}

Conflict of Interest The authors declare that they have no conflict of interest.

Code Availability Not applicable.

\section{References}

1. Oluwole SF, Ali AO, Adu A et al (2003) Impact of a cancer screening program on breast cancer stage at diagnosis in a medically underserved urban community. J Am Coll Surg 196(2):180-188

2. Freeman HP, Wasfie TJ (1989) Cancer of the breast in poor black women. Cancer. 63(12):2562-2569

3. International Agency for Research on Cancer (2019) The global cancer observatory: Nigeria. http://gco.iarc.fr/today/data/ factsheets/populations/ 566-nigeria-fact-sheets.pdf

4. Chidebe RC, Orjiakor CT, Pereira I, Ipiankama SC, Lounsbury DW, Moraes FY (2019) Navigating prostate cancer control in Nigeria. Lancet Oncol 20(11):1489-1491

5. Federal Ministry of Health (2018) Nigeria National Cancer Control Plan (NCCP) (2018-2022). https://www.iccpportal.org/system/ files/plans/NCCP_Final\%20\%5B1\%5D.pdf

6. National Health Service Digital NHS Digital (2019) Breast Screening Programme. https://digital.nhs.uk/data-and-information/ publications/statistical/breast-screening-programme

7. National Institute for Health and Care Excellence (2017) Breast screening. https://cks.nice.org.uk/breast-screening\#!scenario

8. Knapp GC, Tansley G, Olasehinde O et al (2019) Mapping geospatial access to comprehensive cancer care in Nigeria. JCO Global Oncol 5:1-8

9. Dodo AM, Sykes P, Powell C (2016) Exploring the barriers to breast and cervical cancer screening in Nigeria: a narrative review. Afr J Reprod Health 20(4):89-98

10. Okoronkwo IL, Ejike-Okoye P, Chinweuba AU, Nwaneri AC (2015) Financial barriers to utilization of screening and treatment services for breast cancer: an equity analysis in Nigeria. Niger $\mathrm{J}$ Clin Pract 18(2):287-291

11. Modibbo FI, Dareng E, Bamisaye P et al (2016) Qualitative study of barriers to cervical cancer screening among Nigerian women. BMJ Open 6(1):e008533

12. Chidebe RCW, Tauriainen L, Orjiakor TC et al (2018) Patient navigation: breaking the barriers of care while empowering patients to fight cancer in Nigeria. JCO Global Oncol 244s 
13. Kashima K, Phillips S, Harvey A et al (2018) Efficacy of the competency-based oncology patient navigator training. J Oncol Navig Survivorship 9(2):519-524

14. Pratt-Chapman ML, Willis A, Masselink L (2015) Core competences for oncology patient navigators. J Oncol Navig Survivorship 6(2):16-21

15. Onoka CA, Onwujekwe OE, Uzochukwu BS, Ezumah NN (2013) Promoting universal financial protection: constraints and enabling factors in scaling-up coverage with social health insurance in Nigeria. Health Res Policy Syst 11(1):20

16. Agha AA, Onalu CE, Ekoh PC et al (2020) Utilization of patient navigation model in eliminating the speed bumps to quality healthcare delivery in Nigeria. J Soc Work Dev Soc 2(1)

Publisher's Note Springer Nature remains neutral with regard to jurisdictional claims in published maps and institutional affiliations. 